\title{
Reflets
}

Revue ontaroise d'intervention sociale et communautaire

\section{Des pratiques à notre image : défis et ressources}

\section{Madeleine Dubois et Nérée St-Amand}

Volume 1, numéro 1, printemps 1995

Des pratiques à notre image : défis et ressources

URI : https://id.erudit.org/iderudit/026051ar

DOI : https://doi.org/10.7202/026051ar

Aller au sommaire du numéro

Éditeur(s)

Reflets : Revue ontaroise d'intervention sociale et communautaire

ISSN

1203-4576 (imprimé)

1712-8498 (numérique)

Découvrir la revue

Citer ce document

Dubois, M. \& St-Amand, N. (1995). Des pratiques à notre image : défis et ressources. Reflets, 1(1), 1-4. https://doi.org/10.7202/026051ar

Tous droits réservés (C) Reflets : Revue ontaroise d'intervention sociale et communautaire, 1995
Ce document est protégé par la loi sur le droit d'auteur. L'utilisation des services d'Érudit (y compris la reproduction) est assujettie à sa politique d'utilisation que vous pouvez consulter en ligne.

https://apropos.erudit.org/fr/usagers/politique-dutilisation/ 


\section{Des pratiques à notre image:}

\section{défis et ressources}

É crire est, qu' on le veuille ou non, un verbe politique... l'éciture n'engendre sans doute pas l'identité collective, mais éluade ses lieux d'origine François Paré

\section{M adeleine $D$ ubois et $N$ érée St-A mand}

C o-rédacteurs

Dans la création de ce premier numéro, le verbe écrire a été conjugué à tous les temps et a donné lieu à un foisemment d'activités telles que l'entraide, les échanges, les confrontations, les réflexions individuelles et les débats collectifs. C es activités ont alimenté les efforts d'appropriation et de définition des pratiques sociales et communautaires nous caractérisant... pratiques tant communautaires qu'académiques et institutionnelles, pratiques des intervenantes et des intervenants, des étudiantes et des étudiants des diverses régions, bref, pratiques de personnes engagées à de multiples niveaux dans des réflexions sur le sens et l'orientation de l'intervention sociale en 0 ntario français.

Les articles de ce numéro décrivent et illustrent certaines particularités de pratiques liées au contexte social, politique, économique et historique de notre province et ancrées dans les divers milieux franco-ontariens. C elles-ci se développent et se définissent en fonction des ressources et des problèmes spécifiques à une variété de régions rurales et urbaines aux quatre coins de la province. Elles tentent d'apporter des éléments de réponses aux défis propres à chacun de ces milieux. Elles sont aussi fort originales, fruit de la créativité de personnes et d'organismes cherchant à comprendre, à définir des besoins et à y répondre de la façon la plus pertinente possible, compte tenu des nombreuses contraintes liées, notamment, à la pratique en milieu minoritaire. 
Plusieurs articles de ce numéro illustrent cette diversité et cette originalité: le modèle du SIEN (Services intégrés pour les enfants du nord) présenté par $M$ arieTurcotte et s'adressant aux familles du vaste territoire du nord-est ontarien, et le modèle de coordination «supra agences» esquissé par A lain Poirier, et s'adressant aux famillesfrancophones de la région urbaine d' $\mathrm{O}$ ttawa $\mathrm{C}$ arleton, ne sont que deux exemples de modèles créatifs de prestation de services pour une population dont les besoins spécifiques sont influencés, entre autres, par leur situation géographique et démographique.

L'hétérogénéité grandissante de la population franco-ontarienne, suite à l'arrivée de personnes originaires d'A frique, de $\mathrm{H}$ aïti ou d'ailleurs, nous invite aussi à une réflexion sur la décentration, afin de mieux reconnaitre et nommer nos particularités culturelles, et d'être en mesure de développer des pratiques qui tiennent compte de nos diversités. L'article de $\mathrm{M}$ athilde $\mathrm{N}$ duwimana et d'A lice $H$ ome, et celui signé par Lamine $D$ iallo et $M$ arge $R$ eitsma Street, proposent des exemples concrets de défis suscités par le renouvellement et l'adaptation de nos pratiques en fonction de ces nouvelles réalités. De son côté, le concept de décentration, abordé par Anselme M vilongo-T sala, offre des pistes de questionnement et de réflexion qui nous interpellent, tout en se voulant une source d'inspiration dans la mise en oeuvre de pratiques répondant à des besoins variés.

Le thème de ce premier numéro de la revue $R$ eflets, «des pratiques à notre image», a aussi été choisi dans le but de faire émerger les caractéristiques qui font partie de notre histoire et qui se tissent dans les pratiques que nous inventons ou développons. Comme il existe peu d'écrits sur la création et la mise en oeuvre des services sociaux, communautaires et de santé en français en 0 ntario, il est intéressant de constater, dans cette série d'articles, l'émergence de thèmes liés à notre histoire, et toujours d'actualité dans nos préoccupations et nos défis quotidiens.

$D$ ans son analyse des liens entre l'histoire politique et économique desFranco- 0 ntarienneset $F$ ranco- 0 ntariens et le développement des services en français en $O$ ntario, $D$ avid W elch constate que certains des nôtres, plus vulnérables, ont été fréquemment laissés pour compte dans les nombreuses revendications pour l'obtention 
de nos droits. Ce processus de fragilisation, voire d'exclusion de certains membres de nos communautés, demeure une préoccupation, comme en fait état l'article de $M$ arc $M$ olgat, qui relate les trajectoires de vie de jeunes sans-abri francophones de la région d'O ttawa-C arleton.

Certains modèles d'intervention, axés sur les valeurs et sur l'idéologie d'une culture ou de groupes dominants, peuvent aussi contribuer à la marginalisation, voire à l'oppression de personnes ou de groupes minoritaires. Par le biais d'une analyse du discours de thérapeutes intervenant auprès des familles franco-ontariennes, M irelle G élinasillustre comment certainsmodèles de pratique contribuent à la marginalisation de certaines couches de la population; elle souligne de plus l'importance de pratiques thérapeutiques conscientisantes dans le travail avec des groupes minoritaires.

Ce souci de nommer et de faire face aux problèmes exprimés par les gens constitue par ailleurs un autre fil conducteur soustendant les articles de ce numéro. Les problèmes de pauvreté, d'isolement, de chômage et de manque de formation, liés à des questions d'identité et de revendication de droits, constituent autant de défis pour toute personne intervenant en 0 ntario français. Le désir de venir en aide aux personnes plus marginalisées se dégage de nombreux articles de la rubrique «aux quatre coins». $\mathrm{N}$ ous y présentons des services, des programmes et des initiatives francophones dans les domaines de la promotion de la santé, des services sociaux scolaires, de la violence conjugale, de l'agression sexuelle et de la santé mentale.

Voilà autant de raisons pour lesquelles ce numéro se veut une source d'inspiration. En effet, se familiariser avec ce qui se passe àWelland, à $\mathrm{H}$ amilton, à Pembroke ou à $\mathrm{H}$ earst peut susciter des dialogues entre les diverses régions de la province, aptes à inspirer et enrichir l'ensemble de nos pratiques. $N$ ous espérons que ces réponses locales, cesinitiatives émergeant de divers milieux pourront aider celles et ceux qui sont à la recherche de nouvelles pistes pour faire face aux défis de l'heure.

Décidément, ce premier numéro de $R$ eflets marque la concrétisation d'un défi que nous avonstenté de relever en composant avec les contraintes auxquelles nous avions à faire face. Cette 
expérience s'est avérée particulièrement enrichissante, grâce au dialogue et au travail d'équipe entre les auteures et auteurs et les membres de l'équipe de rédaction. N ous voulons souligner la collaboration de toutes et de tous, et plus encore, la fierté souvent exprimée à l'idée de contribuer à cette revue, à notre revue. La réponse des gens était enthousiaste, même lorsque les délais étaient très courts.

Ce travail de collaboration entre le comité de rédaction, le comité de direction, l'équipe responsable du numéro, les personnes qui ont soumis des textes, celles qui ont agi comme arbitres et celles qui ont corrigé les épreuves, se dégage comme un fait marquant de ce premier numéro. En plus de toutes ces personnes qui ont mis la main à la pâte, soulignons la contribution des institutions par l'offre d'appui financier ou de ressources humaines, sans oublier le travail en coulisse de Sonia $C$ adieux, qui a colligé tous les articles, refait maintes corrections et vérifications, et offert un appui administratif d'une efficacité exemplaire. $R$ eflets est sans contredit le fruit d'un projet collectif, auquel nous sommes fiers de participer.

$N$ ous espérons que grâce à cet instrument de communication, les pratiques d'ici seront davantage connues, et surtout reconnues. N otre objectif est que ce moyen de communiquer vous soit utile, peu importe l'endroit où vous oeuvrez. Et puisque cette revue se veut un endroit d'échange, nous attendons vos réactions, commentaires, suggestions, et articles pour les prochains numéros qui porteront sur la santé communautaire et sur la violence.

N ous souhaitons vivement que, par le biais de cette revue, le verbe «écrire» continue de se conjuguer à tous les temps...

Bonne lecture!

\section{Bibliographie}

PAR É, François (1994). «'institution littéraire franco-ontarienne et son rapport à la construction identitaire des Franco- $O$ ntariens», dans $L$ a question identitaire du $C$ anada francophone. R éits, parcours, enjeux, hors lieux, sous la direction de Jocelyne Létourneau, R oger Bernard (coll.), SainteFoy, Les Presses de l'U niversité Laval, p. 61. 